\title{
Anastomose Biliar no Transplante Hepático: Com ou Sem Tubo em T?
}

\author{
Biliary Anastomosis in Liver Transplantation: With or \\ Without T-Tube?
}

\author{
Janine CARMELINO $\rrbracket^{1}$, Susana RODRIGUES ${ }^{1}$, Hugo Pinto MARQUES ${ }^{1}$, Vasco RIBEIRO ${ }^{1}$, Daniel VIRELLA², \\ Marta ALVES ${ }^{2}$, Américo MARTINS ${ }^{1}$, Eduardo BARROSO 1 \\ Acta Med Port 2017 Feb;30(2):122-126 - http://dx.doi.org/10.20344/amp.7287
}

\section{RESUMO}

Introdução: Complicações biliares ocorrem em 10\% - 30\% dos transplantes hepáticos. O objetivo deste trabalho é comparar as incidências dessas complicações nos transplantes hepáticos em que foi ou não utilizado tubo em T na anastomose biliar.

Material e Métodos: Análise de dois grupos de doentes submetidos a transplante hepático entre 2008 e 2012. Consideraram-se os doentes em que o tubo em T foi utilizado (G1) e em que não o foi (G2). Procuraram-se depois modelos explicativos da ocorrência de complicações biliares por regressão logística, incluindo as variáveis identificadas na análise univariável.

Resultados: Estudaram-se 506 doentes consecutivos submetidos a um primeiro transplante hepático $(\mathrm{G} 1=363 ; \mathrm{G} 2=143)$. A incidência global de complicações biliares foi 24,7\% (IC 95\% 21,1 - 28,6): 27,0\% no G1 e 18,9\% no G2 ( $p=0,057)$. As incidências de estenose e de fístula biliar foram tendencialmente mais elevadas em G1: 19,6\% (IC 95\% 15,7-23,8) vs 15,4\% (IC 95\% 10,1 - 22,0) $(p=0,275)$ e $6,6 \%$ (IC 95\% 4,4 - 9,5) vs 2,8\% (IC 95\% 0,9 - 6,6) ( $p=0,091)$. Não se encontraram diferenças estatisticamente significativas nas taxas de colangiopancreatografia retrógrada endoscópica, reoperação e retransplante. Verificaram-se dois óbitos no G1. Não se encontrou associação entre a ocorrência de complicações biliares e os diâmetros das vias biliares ou o tempo de isquemia fria. $\mathrm{O}$ modelo explicativo ajustado à idade do recetor e do dador, e ao diagnóstico de base identifica o uso do tubo em T como aumentando a possibilidade da ocorrência de complicações biliares (AdjOR 1,71; IC 95\% 1,04 - 2,80; $p=0,034$ ).

Discussão e Conclusão: A utilização do tubo em T deve ser uma decisão tomada caso a caso e baseada no julgamento intra-operatório de cirurgiões experientes.

Palavras-chave: Anastomose Cirúrgica; Transplante de Fígado/métodos

\section{ABSTRACT}

Introduction: Biliary complications occur in $10-30 \%$ of liver transplants. The aim of this study was to compare the incidence of these complications in liver transplants when the T-tube was or was not used during the biliary anastomosis.

Material and Methods: Analysis of 2 groups of patients undergoing liver transplantation between 2008 and 2012. Patients were divided considering if the T-tube was used (G1) or if it was not (G2). We sought explanatory models of the occurrence of biliary complications by logistic regression, including the variables identified in the univariate analysis.

Results: We reviewed 506 consecutive patients who underwent a first liver transplant $(\mathrm{G} 1=363, \mathrm{G} 2=143)$. The overall incidence of biliary complications was $24.7 \%(95 \% \mathrm{Cl} 21.1$ to 28.6$): 27.0 \%$ in $\mathrm{G} 1$ and $18.9 \%$ in $\mathrm{G} 2(p=0.057)$. The incidences of stenosis and biliary fistula tended to be higher in $\mathrm{G} 1: 19.6 \%(95 \% \mathrm{Cl} 15.7$ to 23.8$)$ vs $15.4 \%$ (95\% Cl 10.1 to 22.0$)(p=0.275)$ and $6.6 \%(95 \% \mathrm{Cl} 4.4$ to 9.5$)$ vs $2.8 \%(95 \% \mathrm{Cl} 0.9$ to 6.6$)(p=0.091)$. We did not find statistically significant differences in the rates of endoscopic retrograde cholangiopancreatography, reoperation and retransplantation. There were two deaths in $\mathrm{G} 1$. There was no association between the occurrence of biliary complications and the diameters of the biliary tract nor the time of cold ischemia. The explanatory model, adjusted to the recipient and the donor age's and to the initial diagnosis, identifies the use of the T-tube as increasing the possibility of the occurrence of biliary complications (AdjOR $1.71,95 \% \mathrm{Cl} 1.04$ to $2.80 ; p=0.034$ ).

Discussion and Conclusion: The use of the T-tube should be a decision taken on a case-based intraoperative judgment of experienced surgeons

Keywords: Anastomosis, Surgical; Liver Transplantation/methods

\section{INTRODUÇÃo}

A reconstrução biliar continua a ser o "calcanhar de Aquiles' da transplantação hepática e é responsável por taxas de complicação entre 10 e $30 \%$ dos doentes. ${ }^{1}$

As estenoses e fístulas biliares são o tipo de complicação mais frequente no transplante hepático com enxerto de dador cadáver, e ocorrem respetivamente em nove a $12 \%$ e cinco a $10 \%$ dos casos. ${ }^{2}$

A utilização sistemática da coledococoledocostomia sob tubo em $\mathrm{T}$ no transplante hepático proveio da experiência adquirida na cirurgia biliar. Contudo, nos últimos anos o seu uso tem sido progressivamente mais contestado por vários autores, sobretudo em anastomoses com vias biliares de diâmetros semelhantes e bem vascularizadas. , $^{1,4}$

No nosso centro de transplantação, o tipo de anastomose biliar que privilegiamos é a coledococoledocostomia termino-terminal. As principais vantagens desta técnica são permitir preservar o mecanismo de esfíncter e facilitar uma posterior abordagem terapêutica por via endoscópica. ${ }^{5}$

$\mathrm{O}$ uso do tubo em $\mathrm{T}$ tem inúmeras vantagens: permite calibrar e diminuir a pressão na via biliar, monitorizar a quantidade e qualidade da bílis produzida e fazer estudos imagiológicos da árvore biliar. ${ }^{1}$

\footnotetext{
1. Serviço de Cirurgia Geral. Hospital de Curry Cabral. Centro Hospitalar de Lisboa Central. Lisboa. Portugal.

2. Gabinete de Análise Epidemiológica e Estatística. Centro de Investigação. Centro Hospitalar de Lisboa Central. Lisboa. Portugal.

$\bowtie$ Autor correspondente: Janine Carmelino. janine.carmelino@chleiria.min-saude.pt

Recebido: 05 de dezembro de 2015 - Aceite: 28 de novembro de 2016 | Copyright @ Ordem dos Médicos 2017
} 
Contudo, existem também desvantagens: maior desconforto do doente, possível porta de entrada para microorganismos, necessidade de um segundo internamento para a sua remoção e risco de fístula no orifício de entrada do tubo, com consequente risco de peritonite biliar. ${ }^{1}$

Vários autores têm analisado esta questão e tentado esclarecer esta controvérsia. Os resultados têm sido variados e dissonantes. ${ }^{1-9}$

No nosso Centro, a decisão de utilizar o tubo em T é avaliada caso a caso. Procedemos à sua utilização sistemática nas reconstruções biliares em que o risco de complicações é previsivelmente superior: vias biliares de pequeno calibre (menor que 7 milímetros), nos retransplantes e nos casos de enxertos marginais, ou com um tempo de isquemia fria prolongada.

O objetivo deste estudo é comparar a incidência de complicações biliares nos doentes em que foi ou não utilizado tubo em T durante o transplante hepático.

\section{MATERIAL E MÉTODOS}

Os autores procederam à análise dos processos clínicos dos 577 doentes submetidos a transplante hepático, num único centro de transplantação, entre 2008 e 2012 (cinco anos).

Foram considerados critérios de exclusão: casos em que o receptor tinha idade inferior a 18 anos, retransplantes hepáticos, transplantes com enxertos 'small for size' e transplantes cuja reconstrução biliar consistiu em anastomose bilio-digestiva.

Após análise das coortes históricas dos 506 doentes incluídos no estudo, foram considerados dois grupos: Grupo 1 (G1), em que o tubo em T foi utilizado e Grupo 2 (G2) em que o tubo em $T$ não foi utilizado.

A decisão de utilizar ou não tubo em $\mathrm{T}$ tinha resultado da avaliação intraoperatória do cirurgião.

Recolhemos dos processos clínicos, os dados relativos às variáveis que depois analisámos: principais características do dador e recetor, diagnóstico base que levou ao transplante hepático, resultados pós operatórios globais, tipos de complicações biliares ocorridas e tipos de terapêutica utilizada na sua resolução.

Aos doentes do G1 foi feita colangiografia entre o sétimo e o $10^{\circ}$ dia pós operatório. Estes doentes tiveram alta com o tubo em T clampado. O mesmo foi removido após nova colangiografia, seis meses após o transplante.

Tabela 1 - Principais características dos doentes

\begin{tabular}{|c|c|c|c|}
\hline Característica & $\begin{array}{c}\text { Grupo } 1 \\
n=363\end{array}$ & $\begin{array}{c}\text { Grupo } 2 \\
n=143\end{array}$ & Valor $p$ \\
\hline Idade do dador (anos) & $46,8(17,4)$ & $50,4(15,3)$ & 0,023 \\
\hline Tempo de isquemia fria (minutos) & $384(64,5)$ & $382(67,5)$ & 0,840 \\
\hline Diferença de diâmetro da via biliar principal (milímetros) & $2,2(2,3)$ & $1,6(1,8)$ & 0,093 \\
\hline Idade do recetor (anos) & $48,5(12,6)$ & $47,2(13,1)$ & 0,312 \\
\hline $\begin{array}{l}\text { Sexo do receptor } \\
\text { - Masculino } \\
\text { - Feminino }\end{array}$ & $\begin{array}{l}255(70 \%) \\
108(30 \%)\end{array}$ & $\begin{array}{l}84(59 \%) \\
59(41 \%)\end{array}$ & 0,013 \\
\hline
\end{tabular}

Grupo 1: Reconstrução biliar com tubo em T; Grupo 2: Reconstrução biliar sem tubo em T. Valores em média (desvio padrão)
Consideraram-se sintomas suspeitos de fuga biliar após remoção de tubo em $\mathrm{T}$, febre e sinais de irritação peritoneal nas primeiras 24 horas após a colangiografia. A fuga foi sempre confirmada imagiologicamente.

A hipótese diagnóstica de colangite foi colocada na presença de febre, aumento dos parâmetros inflamatórios e de colestase.

A presença de estenose biliar foi sempre confirmada por imagiologia e endoscopia.

O tempo médio (desvio padrão) de acompanhamento após transplante hepático foi de 1174 (32) dias. Os doentes foram reavaliados em consultas mensais durante os primeiros seis meses após transplante, trimestrais nos 18 meses seguintes, e semestrais a partir dessa data.

A comparação de proporções foi feita com o teste exato de Fisher ou com o teste Qui-quadrado. A precisão das taxas de incidência foi dada pelos intervalos de confiança de 95\% (IC 95\%).

$\mathrm{Na}$ análise univariável identificaram-se as variáveis elegíveis para elaborar, por regressão logística, modelos explicativos da ocorrência de complicações biliares.

Foi utilizado o software SPSS 22.0 (SPSS for Windows, Rel. 22.0.1. 2013. SPSS Inc., Chicago, II, EUA)

\section{RESULTADOS}

Dos 506 doentes estudados, 363 foram submetidos a reconstrução biliar com tubo em T (Grupo 1) e 143 a reconstrução biliar sem tubo em T (Grupo 2).

As principais características dos dois grupos são apresentadas na Tabela 1. Apenas a idade média do dador (mais elevada no Grupo 2) e o sexo do receptor (maior percentagem de doentes do sexo masculino no Grupo 1) eram significativamente diferentes nos dois grupos.

Os dados relativos aos grupos de diagnóstico base que levaram ao transplante hepático são apresentados na Tabela 2. Procedendo a uma análise bivariável e aplicando o teste Qui-quadrado encontrámos algumas diferenças estatisticamente significativas entre os dois grupos. A opção de utilizar tubo em $T$ foi mais frequente nos doentes transplantados por doenças hepáticas não virais com cirrose e por tumores, e foi menos frequente nos casos de doenças metabólicas.

Após comparação dos resultados pós operatórios globais nos dois grupos (morbilidade global e a morbilidade por causas não biliares) não encontrámos diferenças 
estatisticamente significativas (valor $p=0,154$ e 0,083 respetivamente) (Tabela 3).

A incidência de complicações trombóticas e hemorrágicas nos dois grupos também não difere de forma estatisticamente significativa (valor $p=0,065$ e valor $p=0,145$ respetivamente) (Tabela 3 ).

A taxa de complicações biliares foi mais elevada nos doentes do Grupo 1 (27\% vs 18,9\%; valor $p=0,057$ ). Detetámos também uma tendência para que os três principais tipos de complicações biliares (estenoses, fístulas biliares e bilomas) sejam mais frequentes nos doentes do Grupo 1 (Tabela 3).

Relativamente aos procedimentos utilizados para resolução dessas complicações houve uma percentagem maior de doentes do grupo um com necessidade de internamento para medidas conservadoras $(13,5 \%$ vs $4,9 \%$; valor $p=0,050)$. Não encontrámos diferença significativa entre os dois grupos quanto ao número de colangiopancreatografias retrógradas endoscópicas (valor $p=0,123$ ), reintervenções cirúrgicas (valor $p=1,000$ ) ou retransplantes efetuados para tratar complicações biliares ( valor $p=0,770$ ) (Tabela 3).

Os dois casos de óbito verificados no Grupo 1 ocorreram em doentes propostos para retransplante hepático por colangites de repetição, que faleceram devido a choque he- morrágico refratário após esse retransplante. (Tabela 3).

Nos doentes do Grupo 1, o tempo médio (desvio padrão) até à remoção do tubo em T foi de 9,3 $(3,7)$ meses.

Dos 98 casos de complicações biliares, sete $(7,1 \%)$ ocorreram durante o segundo internamento para remoção do tubo em T. Quatro desses doentes tiveram dor abdominal com necessidade de prolongamento do internamento para vigilância e tratamento conservador, dois tiveram fuga biliar, com necessidade de tratamento endoscópico, e um teve peritonite biliar com necessidade de relaparotomia urgente.

Na análise univariável não se encontrou associação entre a taxa de complicações biliares e o diâmetro das vias biliares, nem entre a taxa de complicações biliares e o tempo de isquemia fria.

Da análise de regressão logística, concluímos que o uso de dreno biliar parece aumentar $59 \%$ a possibilidade de ocorrência de complicações biliares (OR 1,59, IC 95\% $0,98-2,56 ; p=0,058)$

Quando o modelo explicativo foi ajustado às idades do recetor e do dador e ao diagnóstico de base, o uso do tubo em $\mathrm{T}$ foi identificado como aumentando $71 \%$ a possibilidade de ocorrência de complicações biliares (AdjOR 1,71; IC 95\% 1,04 - 2,80; $p=0,034$ ) (Tabela 4).

Tabela 2 - Distribuição da amostra pelos grupos de diagnósticos base que motivaram o transplante hepático

\begin{tabular}{lccc}
\hline Diagnóstico base & $\begin{array}{c}\text { Grupo 1 } \\
\mathbf{n = 3 6 3}\end{array}$ & $\begin{array}{c}\text { Grupo 2 } \\
\mathbf{n = 1 4 3}\end{array}$ & Valor $\boldsymbol{p}$ \\
\hline Cirrose viral & $30(8,3 \%)$ & $16(11,2 \%)$ & 0,200 \\
Hepatite fulminante & $32(8,8 \%)$ & $7(4,9 \%)$ & 0,200 \\
Doenças hepáticas não virais c/cirrose & $100(27,5 \%)$ & $38(26,6 \%)$ & $<0,001$ \\
Tumores & $116(32,0 \%)$ & $32(22,4 \%)$ & $<, 001$ \\
Doenças metabólicas & $85(23,4 \%)$ & $50(35,0 \%)$ & 0,003 \\
Teste Qui-quadrado & & Valor $p=0,022$ & \\
\hline
\end{tabular}

Teste Qui-quadrado. Grupo 1: Reconstrução biliar com tubo em T; Grupo 2: Reconstrução biliar sem tubo em T. Valores absolutos (percentagem).

Tabela 3 - Resultados pós operatórios globais e formas de tratamento das complicações biliares

\begin{tabular}{lccc}
\hline & $\begin{array}{c}\text { Grupo } 1 \\
\mathbf{n = 3 6 3}\end{array}$ & $\begin{array}{c}\text { Grupo 2 } \\
\mathbf{n}=143\end{array}$ & Valor $\boldsymbol{p}$ \\
\hline Morbilidade global & $228(62,8 \%)$ & $80(55,9 \%)$ & 0,154 \\
Complicações não biliares & $196(54,0 \%)$ & $65(45,5 \%)$ & 0,083 \\
Complicações trombóticas & $41(11,3 \%)$ & $8(5,6 \%)$ & 0,065 \\
Complicações hemorrágicas & $42(11,6 \%)$ & $10(7,0 \%)$ & 0,145 \\
Complicações biliares & $98(27,0 \%)$ & $27(18,9 \%)$ & 0,057 \\
Fístula & $24(6,6 \%)$ & $4(2,8 \%)$ & 0,091 \\
Estenose & $71(19,6 \%)$ & $22(15,4 \%)$ & 0,275 \\
Biloma & $3(0,8 \%)$ & $1(0,7 \%)$ & 1,000 \\
Mortalidade global & $33(9,1 \%)$ & $8(5,6 \%)$ & 0,194 \\
Tratamento conservador & $49(13,5 \%)$ & $7(4,9 \%)$ & 0,050 \\
Tratamento endoscópico & $75(20,7 \%)$ & $21(14,7 \%)$ & 0,123 \\
Re-intervenção cirúrgica & $9(2,5 \%)$ & $3(2,1 \%)$ & 1,000 \\
Re-transplante hepático & $11(3,0 \%)$ & $3(2,1 \%)$ & 0,770 \\
\hline
\end{tabular}

Grupo 1: Reconstrução biliar com tubo em T; Grupo 2: Reconstrução biliar sem tubo em T. Teste Qui-quadrado. Valores absolutos (percentagem). 
Tabela 4 - Modelo explicativo da ocorrência de complicações biliares. Análise de regressão logística, ajustada às idades do receptor e do dador e ao diagnóstico de base.

\begin{tabular}{llccc}
\hline & Valor $\boldsymbol{p}$ & OR & \multicolumn{2}{c}{ IC $95 \%$} \\
\hline Tubo em T & 0,034 & 1,708 & 1,042 & 2,801 \\
Idade do recetor & 0,386 & 1,009 & 0,988 & 1,031 \\
Idade do dador & 0,096 & 1,011 & 0,998 & 1,023 \\
Cirrose viral & 0,845 & 1,085 & 0,478 & 2,460 \\
Hepatite fulminante & 0,862 & 1,078 & 0,465 & 2,500 \\
Tumores & 0,989 & 0,995 & 0,508 & 1,951 \\
Doenças metabólicas & 0,634 & 0,843 & 0,416 & 1,706 \\
\hline
\end{tabular}

OR: Odds ratio; IC: Intervalo de confiança

\section{DISCUSSÃO e CONCLUSÃO}

Neste estudo de efetividade com 506 doentes submetidos a transplante hepático, identificou-se uma tendência para ocorrerem mais complicações biliares nos doentes com reconstrução biliar sob tubo em $\mathrm{T}$.

Estes nossos resultados contrariam as conclusões de vários estudos publicados na última década.

Na metanálise de Huang et al, que inclui 1608 doentes, o uso do tubo em T é apontado como diminuindo a incidência de estenoses, sem contudo aumentar a incidência de outras complicações biliares. ${ }^{6}$

Sun et al avaliaram os resultados de 15 estudos e concluíram que a incidência de estenoses biliares é significativamente maior nos doentes cuja anastomose foi feita sem tubo em T ( $p<0,001$; OR = 0,49; IC 95\%: 0,34 - 0,69). ${ }^{7}$

López-Andújar et al no seu ensaio clínico prospetivo aleatorizado que incluiu 187 doentes, detetaram uma redução do risco absoluto de ocorrência de estenoses biliares de 12\% (IC 95\% 4 - 20) com a utilização do tubo em T. ${ }^{1}$

A análise retrospetiva de 95 casos feita por Benítez Cantero et al também concluiu que a estenose biliar era mais frequente quando o tubo em T não tinha sido utilizado. ${ }^{8}$

Sascha Weiss et al analisaram de forma prospetiva aleatorizada 194 doentes e consideram o uso do tubo em T uma medida segura e que permite diminuir o número de procedimentos invasivos após o transplante hepático. ${ }^{9}$

Conclusões contrárias resultaram da metanálise de Paes-Barbosa et al. Estes autores sugerem que a coledococoledocostomia deve ser efetuada sem tubo em $T$ no caso de ser termino-terminal mas com utilização de drenagem se for latero-lateral. ${ }^{5}$

Os resultados da metanálise publicada por Sotiropoulos et al (1027 doentes) são mais próximos dos obtidos no nosso estudo. Também neste estudo, o uso sistemático de tubo em T levou a um aumento da morbilidade pós operatória, do número de procedimentos radiológicos e dos dias de internamento. ${ }^{2}$

Também no artigo de revisão de Carina Riediger et al, o uso sistemático do tubo em T é contestado. Na metanálise com 639 doentes, só 17 (2\%) beneficiaram do uso de tubo em T para prevenir uma estenose biliar. Partilhamos também a opinião destes autores de que a monitorização não invasiva da função do enxerto pode ser feita através da determinação do fator $\mathrm{V}$ ou da taxa de eliminação do verde de indocianina. ${ }^{3}$

Acreditamos serem pontos fortes do estudo que apresentamos, a grande dimensão da amostra e o facto de se tratar de um estudo realizado num único centro de transplantação, o que permite diminuir a heterogeneidade frequentemente presente nos estudos multicêntricos.

As associações encontradas entre a utilização do tubo em T e a ocorrência de complicações biliares incluem variáveis objetivas e robustas. O fato de se manterem com o ajustamento na análise multivariável reforça a sua credibilidade.

No entanto, acreditamos que a generalização destes resultados deve ser feita com prudência, pois reconhecemos que o estudo apresenta limitações importantes.

A decisão de utilizar ou não tubo em T resultou da avaliação intraoperatória do cirurgião, não foi aleatória, foi independente do protocolo de investigação e baseou-se em algumas variáveis não disponíveis num estudo observacional e com carácter documental como este.

O tubo em $\mathrm{T}$ foi utilizado para proteger as anastomoses biliares nos casos mais complexos (vias biliares de pequeno calibre ou submetidas a um tempo de isquemia fria prolongado), o que resultou num inevitável viés de seleção e na definição de dois grupos assimétricos.

Embora reconheçamos que o estudo não permite concluir de forma inequívoca se a utilização do tubo em T confere ou não benefício na prevenção de complicações biliares, defendemos que os resultados da análise multivariável, ajustada ás idades do dador e do recetor, e ao diagnóstico que motivou o transplante hepático, permite pôr em causa a sua utilização sistemática em todas as anastomoses biliares.

Tal como defendido por López-Andújar et al os dados que obtivemos levam-nos a considerar fundamental identificar os doentes a quem a utilização de tubo em $T$ não confere qualquer vantagem em termos de prevenção de complicações biliares, diminuindo assim o risco de morbilidade associada ao seu uso.

Nesta série, foi detetada uma tendência para a ocorrência de mais complicações biliares no grupo de doentes em que foi utilizado tubo em $\mathrm{T}$.

Contudo, tendo em conta as limitações deste estudo, a nossa conclusão é que o tipo de reconstrução biliar no transplante hepático deve ser uma decisão tomada após análise cuidadosa caso a caso. A utilização do tubo em T pode ser dispensada quando as vias biliares do dador e recetor têm diâmetros semelhantes e são bem vascularizadas.

Mantemos por isso uma postura semelhante á de López-Andújar et al, e utilizamos sempre o tubo em $\mathrm{T}$ nos casos em que existe uma grande diferença de calibre entre a via biliar do enxerto e do recetor, sobretudo quando um dos dois diâmetros é inferior a 7 milímetros. ${ }^{1}$

O julgamento intra-operatório de cirurgiões com experiência, pode evitar o uso de tubo em T em doentes com baixo risco de complicações. 


\section{PROTECÇÃO DE PESSOAS E ANIMAIS}

Este estudo foi aprovado pela Comissão de Ética do Centro Hospitalar Lisboa Central. Os procedimentos seguidos estavam de acordo com a Declaração de Helsínquia da Associação Médica Mundial.

\section{CONFIDENCIALIDADE DOS DADOS}

Os autores declaram ter seguido os protocolos do seu centro de trabalho acerca da publicação de dados.

\section{REFERÊNCIAS}

1. López-Andújar R, Orón EM, Carregnato AF, Suárez FV, Herraiz AM, Rodríguez FS, et al. T-tube or no T-tube in cadaveric orthotopic liver transplantation: the eternal dilemma: results of a prospective and randomized clinical trial. Ann Surg. 2013;258:21-9.

2. Gastaca M. Biliary complications after orthotopic liver transplantation: a review of incidence and risk factors. Transplant Proc. 2012;44:1545-9.

3. Sotiropoulos GC, Sgourakis G, Radtke A, Molmenti EP, Goumas K, Mylona S, et al. Orthotopic liver transplantation: T-tube or not T-tube? Systematic review and meta-analysis of results. Transplantation. 2009;87:1672-80.

4. Riediger C, Müller MW, Michalski CW, Hüser N, Schuster T, Kleeff J, et al. T-Tube or no T-tube in the reconstruction of the biliary tract during orthotopic liver transplantation: systematic review and meta-analysis. Liver Transpl. 2010;16:705-17.

5. Paes-Barbosa FC, Massarollo PC, Bernardo WM, Ferreira FG, Barbosa FK, Raslan M, et al. Systematic review and meta-analysis of biliary reconstruction techniques in orthotopic deceased donor liver

\section{CONFLITOS DE INTERESSE}

Os autores declaram não terem qualquer conflito de interesse relativamente ao presente artigo.

\section{FONTES DE FINANCIAMENTO}

Os autores declaram não ter recebido subsídios ou bolsas para a elaboração do artigo.

transplantation. J Hepatobiliary Pancreat Sci. 2011;18:525-36.

6. Huang WD, Jiang JK, Lu YQ. Value of T-tube in biliary tract reconstruction during orthotopic liver transplantation: a meta-analysis. J Zhejiang Univ Sci B. 2011;12:357-64.

7. Sun N, Zhang J, Li X, Zhang C, Zhou X, Zhang C. Biliary tract reconstruction with or without T-tube in orthotopic liver transplantation: a systematic review and meta-analysis. Expert Rev Gastroenterol Hepatol. 2015;9:529-38.

8. Benítez Cantero JM, Costán Rodero G, Montero Álvarez JL, Ayllón Terán MD, Naveas Polo C, Fraga Rivas E, et al. Biliary complications after liver transplantation using side-to-side Choledochocholedochostomy reconstruction with or without T-tube. Transplant Proc. 2012;44:2098-9.

9. Weiss S, Schmidt SC, Ulrich F, Pascher A, Schumacher G, Stockmann $M$, et al. Biliary reconstruction using a side-to-side choledochocholedochostomy with or without T-tube in deceased donor liver transplantation: a prospective randomized trial. Ann Surg. 2009;250:766-71. 